\begin{tabular}{c} 
journal homepage: http://ijiemjournal.uns.ac.rs/ \\
International Journal of Industrial \\
Engineering and Management \\
Volume $11 /$ No $4 /$ December $2020 / 263-274$ \\
\hline
\end{tabular}

Original research article

\title{
Realization of PLM application integration with AR technology
}

\author{
J. Duda ${ }^{\mathrm{a}}$, S. Oleszek ${ }^{b}$ \\ a Politechnika Krakowska, Kraków, Polska; \\ b Transition Technologies PSC sp. z o.o., Łódź, Polska
}

\section{A B STRACT}

The Augmented Reality (AR) can be used to simulate and improve production processes in a virtual manufacturing system before their physical implementation. These techniques make it possible to connect the real world with the virtual one in real time and in a threedimensional environment. Their effective application to the implementation of the product development phases would significantly limit its subsequent reworking and modifications. The difficulty, however, is to design and integrate AR with virtual production systems. The paper presents a proposal for a technical procedure for the implementation of the integration of a commercial PLM and a proprietary AR system.

\section{ARTICLE INFO}

Article history:

Received November 1, 2020

Revised December 11, 2020

Accepted December 14, 2020

Published online December 22, 2020

Keywords:

Augmented Reality;

CAx;

Product Lifecycle Management

*Corresponding author:

Jan Duda

duda@mech.pk.edu.pl

\section{Introduction}

Augmented reality includes techniques for adding virtual objects to the real world [1]. Augmented reality techniques are therefore defined as those who, in real-time and in a three-dimensional environment, make it possible to connect the real world with the virtual one [2,3]. In other words, the AR system is a system that connects the real world with a computer-generated world.

The Virtual Factory (VF) paradigm is the basis for dealing with the problem of implementing method- ologies to support the design of production systems, processes, simulation, production control, visualization, and others. The ideal implementation of a virtual factory should therefore include the creation of a comprehensive, integral, upgradeable, and scalable representation of the actual factory. It should provide a virtual representation of buildings, resources, processes, and products. The entire factory should be simulated as a continuous and coherent digital model that can be used throughout the entire product lifecycle - from the product idea to the final disassembly of production plants and buildings [4]. 


\section{Management of development phases based on a common model of a product, process, and resource data}

A modern company has to cope with the co-evolution of products, processes, and production systems through strategic and operational management, promotion of engineering changes depending on market requirements, and the dynamics of legislative changes. The essence of these changes is to manage the implementation of development phases based on a common model of a product, process, and resource data (Product, Process, Resource; Figure 1).

One way to implement the idea of a virtual factory is to use PLM class systems [5,6]. They integrate a set of applications supporting product development, which include [7]:

- Product and Portfolio Management (PPM),

- Computer-aided technologies (CAx),

- Manufacturing Process Management (MPM),

- Product Data Management (PDM).

Although these systems provide support for most areas related to the planning and design phases and effectively support product data management and process virtualization, they do not provide certain functionalities relevant to business needs, especially in the area of interoperability $[8,9,10]$. As a result, the whole process of virtual factory realization cannot be carried out with the use of PLM systems in a continuous and coherent way. This is particularly visible in the lack of integration between technologies and data models used in the product design and the production systems design phases [11].

The concept of integration of development phases is discussed in the paper [12].

\section{PLM system architecture integrated with the service performing tasks related to AR technologies}

Modern PLM systems operate according to the client-server model and communication between system clients (the client can be a web browser, another system or system component), and the system is based on HTTP protocol, i.e., in request-response mode. Thus, it is possible to propose a generalized PLM system architecture integrated with modules performing tasks related to VR or AR technologies. The architectural pattern of WWW application for the client-server system in the most general sense assumes organizing the whole system into three layers: data (also referred as persistence layer, includes i.a. database of the system and file server), application (also called processing or business layer) and presentation (client) [13]. At the level of the application layer, where PLM system services realizing business logic are located, an application performing tasks related to the preparation of data for use in VR or AR environments may be integrated. These tasks may include e.g. conversion of 3D data from native engineering formats (.prt, .CATPart, .sldprt, etc.) to a neutral or lightweight format, which enables easier transfer via HTTP protocol and easier display in mobile multimedia applications. They can also be more complex tasks, i.e. processing and preparing 3D models in such a way that they can be used directly in client applications, e.g. in mobile applications or augmented or virtual reality glasses. Response information from the client application, e.g. in the form of metadata, can be sent directly to the PLM system business layer - for further processing - via communication protocol requests. A proposal of a generalized

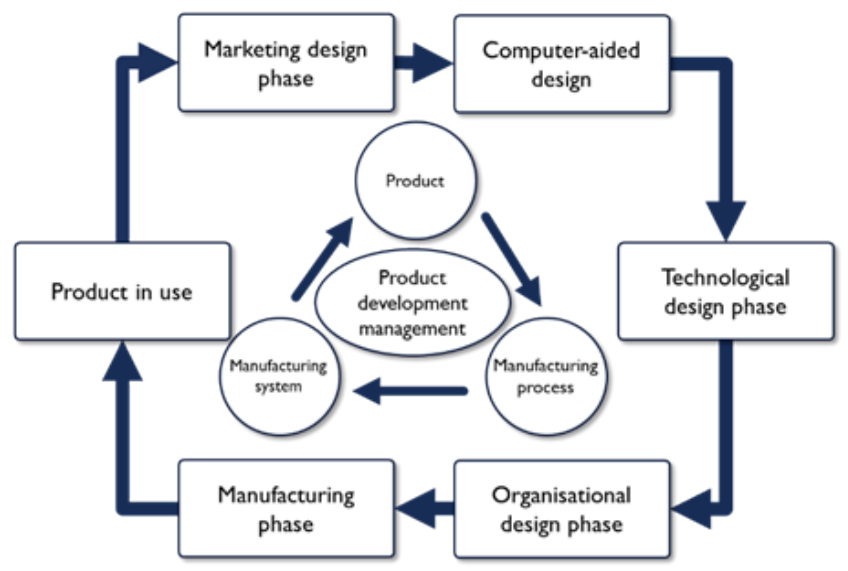

Figure 1. Management of development phases based on a common model of product, process, and resource data 
PLM system architecture integrated with the service performing tasks related to VR and AR technologies is presented in Figure 2.

\section{The proposal of the procedure for the technical implementation of PLM system integration with AR techniques}

This section presents the concept of the procedure for the technical implementation of the integration of the PLM commercial system with the proprietary AR system, where the integration of an advanced CAx class system with a mobile application - in which the AR technology has been applied - was accomplished. This solution enables 3D modeling activities (based on product configurators' techniques) directly in an AR environment. Simultaneously, in the CAx system, a parametric $3 \mathrm{D}$ model is created in a fully automated way [14]. The AR system consists of three components:

(1) A mobile application created in Unity3D environment [15], in which the Vuforia [16] augmented reality library was used,

(2) CAx class system,

(3) An integration module in the form of an application created with the use of the CAx system programming interface in VB.net language, the aim of which is to provide communication with the mobile application and control the automated operations of the CAx system.

The goal of the presented concept of PLM and AR systems integration is:

- implementation of centralized management of standard elements, which are used in the process of modeling and configuration, based on the principles of standards management in PLM system (permission control, versioning, etc.),

- integration of the developed configuration method in the AR environment with a consistent product lifecycle management process,

- implementation of history tracking, status and state management, and versioning of modeled product concepts during their development,

- introducing the possibility of a formalized version approval process for the product created by the customer during the configuration process.

According to the authors' intention, an integrated, uniform environment, including the PLM and AR systems, should allow for all configuration and modeling activities in the AR environment, as developed in [14]. However, all these activities should be performed within a PLM environment. Hence, it is necessary to automate operations such as:

- establishing a connection between the AR and the PLM systems,

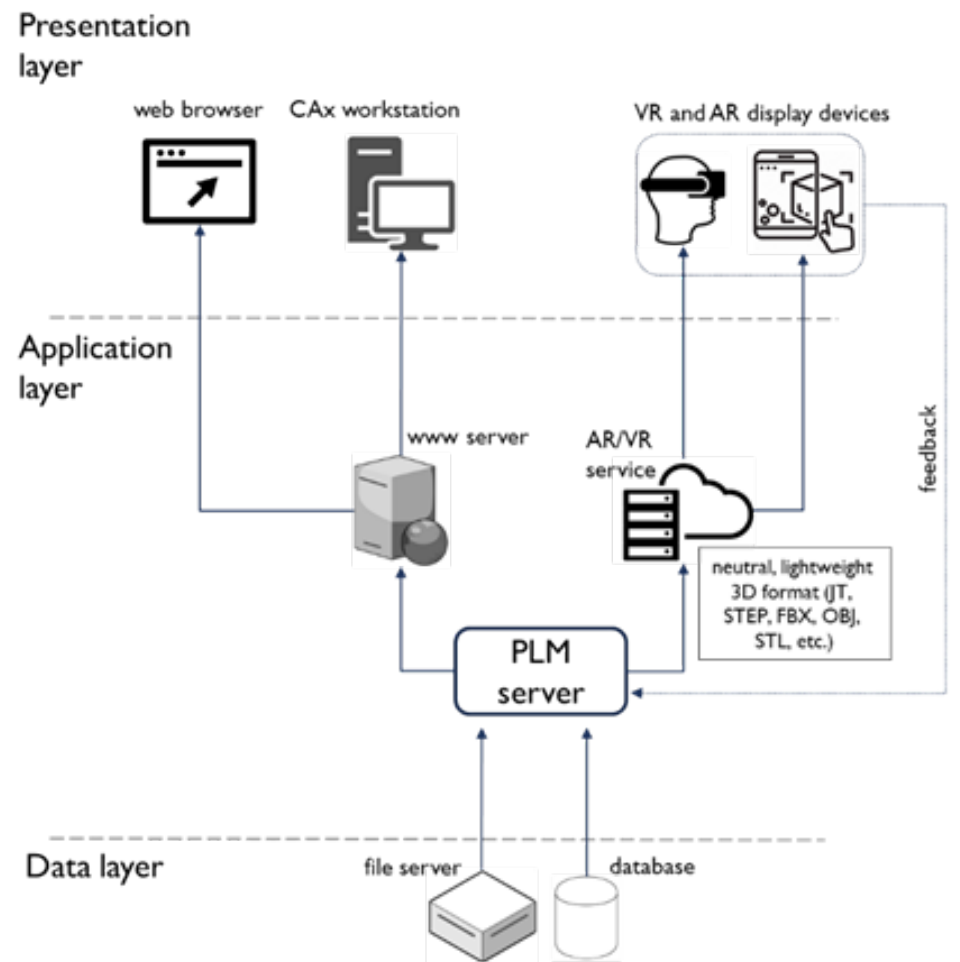

Figure 2. The generalized architecture of the PLM system integrated with the service performing tasks related to VR and AR technologies 
- loading elements used in the configuration process from the PLM system standard library, - saving the 3D model developed in the process of configuration to the development container of the PLM system,

- management of the status and lifecycle state of the $3 \mathrm{D}$ model developed in the configuration process,

- end of session and logout from the PLM system.

The procedure for the implementation of the above-mentioned activities using BPMN notation is discussed in the paper [12].

\section{Technical implementation of PLM system integration with the AR system - the procedure}

This section presents the procedure for the technical implementation of the integration of the proprietary AR system, which was presented in Section 4, with the commercial PLM system - PTC Windchill.

The utilitarian goal of the AR system developed in [14] was to improve the efficiency of the product design process (as illustrated with the example of glass containers) through the active involvement of the (so far passive) user who orders a new product. The aim of the research was an attempt to answer the question of how to shorten the process of developing a new glass container, and thus, how to reflect the customer's requirements in terms of obtained geometrical shape faster and more precisely, and at the same time reduce the costs associated with the project. The expected improvement should result from the correlation of the applied automation techniques used in product configurators and AR computer techniques.

Compared with the conventional method of designing a product (using a CAx system), while using AR environment one can see and test a product in its target context during the process of designing (e.g. on a store shelf or next to the existing products). Consequently, one does not always need a physical prototype, because he or she is allowed to see more and thus, better understand the product.

Therefore, the developed AR system should enable supporting the users in the process of designing glass containers in the following stages of the technical means development process:

1. the process of conceptualization, including:

a. preliminary talks with the client regarding the development and selection of optimal concepts, b. gathering the requirements regarding a new project from the client,

c. selection and comparison of variants of the virtual container models visible in the surroundings of the existing products,

2. design and configuration processes in the AR environment, including:

a. developing a project and carrying out analyses regarding the appearance and shape with the active participation of the client,

b. quickly developing many new variants and presenting them in the real world environment (e.g., surrounded by already existing, real-life glass containers),

c. quickly introduce changes to new or existing 3D models of containers,

d. analysis and evaluation of the developed 3D models in the target context, where the final product will appear, e.g., on a store shelf, on a table, on a shop window, etc.,

e. reconstruction of existing projects for which there is no digital documentation in the form of 3D models,

f. shortening the time of creating a parametric model in the design office,

3. confirmation and faster approval of the requirements and the final project:

a. on the side of the client ordering a new product, including the possibility of presenting the developed project without the need to order physical prototypes,

b. on the manufacturer's side, including the possibility of assessing and carrying out analyzes at various stages of the production process using a 3D model displayed in a given context against the background of the real world.

As indicated in Section 4, the purpose of developing the AR system integration with the PLM environment was to integrate the developed method of supporting the design process in the AR environment with a uniform product lifecycle management process in the PLM environment. Thanks to this integration, it was possible to significantly expand the possibilities and improve the developed method and AR system. According to the authors, the greatest advantages are observed in the functionalities related to the versioning capabilities available in the PLM system, tracking and storing the history of design data, managing their state and status, as well as the ability to manage ac- 
cess and authorizations to modify them. Thanks to the introduction of a centralized and secure, based on the principles underlying the PLM system, the method of managing the design components used in the configuration process, and the product models developed as part of the configuration process, the security of this data has also significantly increased. Integration with the PLM system also enabled the formal approval process of virtual product models developed in the configuration process with the use of an automated workflow.

PTC Windchill system is an advanced PLM class system, which is used in an organization as a collaborative environment. It aims at developing products and manages their lifecycle. PTC Windchill includes many modules and functionalities that comprehensively support the management of all stages of the product development process [17]. Two components of this system were used to carry out the research:

- an advanced CAx class system - Creo Parametric,

- PDM module - PDMLink.

All actions performed by the user in the system during the configuration process are carried out using a mobile device.

During design activities, communication between the AR system and the PLM system is performed in the background through the integration module, which acts as a communication interface in the system. Communication between the mobile device on which the AR system operates and the PLM system is carried out using the TCP/IP protocol, and the data is sent in two formats:

- XML (Extensible Markup Language) - metadata and parameters,

- OBJ - 3D models.

The integrating module, in addition to ensuring data exchange between AR and PLM systems, also acts as a tool that automates design activities in Creo Parametric and activities in the PLM system, mainly related to CAD data management and changing their statuses.

The class of the product that is configured in the described example remains the same as in [14] - they are glass containers.

As it was presented in [12], activities in an integrated AR and PLM environment consist of the following steps:

1. Starting the configuration process,

2. Creating initial product shape in the AR environment,
3. Generating a parametric model in the CAx system,

4. Editing dimensional parameters of the 3D model,

5. Saving the created model in the PLM system,

6. Finishing the process.

The activities in all steps listed above will be presented and further explained later in this section.

\subsection{Starting the configuration process}

In the first step, the user runs the AR application on a mobile device (smartphone or tablet) and begins the design process. At the start of the configuration process in the AR application, the CAx system is launched on the server, and the user is logged into the PLM system in an automated manner. After this operation, a new session is created.

Compared to the solution developed in [14], some significant changes have been introduced in the AR application. In the original version of the application, virtual models were positioned with the use of specially developed markers in the form of colorful images printed on paper. Such a tracking system is classified as marker-based AR. In the configuration process, four markers were used, one for each part of the container model (body, finish, neck, and heel). The markers had to be in the field of view of the camera, and the virtual 3D model that was assigned to it was displayed in the place where the marker was located.

In the current version of the application, a more modern solution has been adopted, which enables the display of virtual models directly on the planes of the real world, eliminating the need for markers. This solution is based on the operation of sensors embedded in mobile devices (including GPS, accelerometer, gyroscope), as well as on more advanced image processing algorithms than in the case of solution based on markers. The new approach uses the newer version of the Vuforia AR engine, and its markerless tracking functionality referred to as Ground Plane [18]. Consequently, the mobile devices that can be used to run the new version of the AR application have to meet the requirements specified on the $\mathrm{Vu}$ foria Developer Portal [19]. Advanced features, such as e.g., Ground Plane, require support for the Positional Device Tracker using Vuforia Fusion [20]. On the list of recommended devices that are enabled via Vuforia Fusion are those, which either support their respective platform provided device tracking technology ARKit/ARCore [21], or they have been calibrated by Vuforia to support Visual-Inertial Si- 
multaneous Localization And Mapping (VISLAM) algorithm [20].

The product configuration process has also been improved. In the original version of the solution, first, it was necessary to select all parts of the model on the dedicated markers, and only then could they be attached to the container body on the main marker. In the current version, all activities are carried out directly on the target model. The user selects the individual parts of the model and sees the result immediately.

The configuration process begins with placing the starting model on the selected plane, which has been recognized by the system and is marked with a white rectangle on the screen of the mobile device (Figure 3).

With the virtual model visible on the screen, the product configuration process can begin. In the presented example, the configuration process will be carried out using a real glass container, which will be used as an example to recreate a virtual model.

\subsection{Creation of the initial product shape in the $A R$ environment}

Design activities in the AR application are carried out using techniques taken from product configurators. This means that in the first stage of the design process, the user obtains the initial shape of the product by selecting predefined parts of the container model, i.e., the finish, neck, body, and heel. To start the configuration process, the user selects the CONFIG button from the user interface of the mobile application. After performing this action, possible configuration options for individual parts of the model are visible on the display screen menu (Figure $4)$.

In the next steps, the user has the option of view-
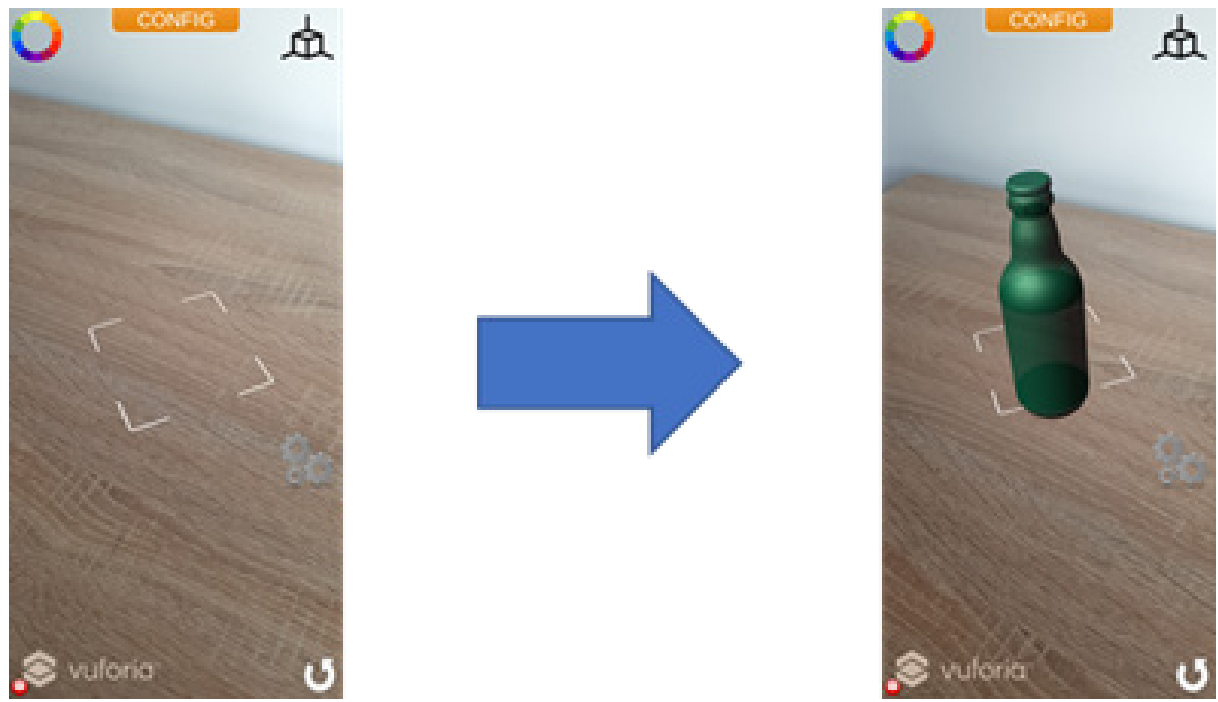

Figure 3. Placing the virtual model on the plane recognized by the AR application algorithm

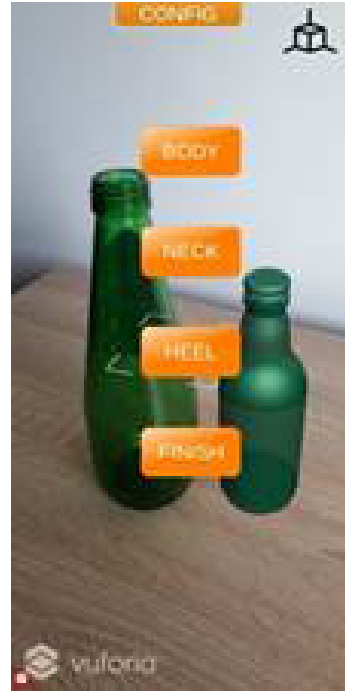

Figure 4. The menu from which the process of selecting individual parts of the 3D model of the glass container begins 
ing all available parts of the container model and comparing the shape of the virtual model with the physical container. The process of viewing a part of the model is shown in Figure 5. After selecting and confirming the initial shape of the virtual container model, the user can move to the second stage of the design process. Then it will be possible to edit the dimensional parameters and obtain the exact shape of the 3D model.

\subsection{Generating a parametric model in the CAx system}

As the initial shape of the $3 \mathrm{D}$ model is approved, an XML file is generated in the AR application, in which information about the parts selected during the configuration process and the dimensional parameters of the obtained model are saved. In the second step, the generated file is sent to the server on which the PLM system is running. The data from the XML file is loaded into the integrating module. Then, on the basis of the information read from the file, the appropriate design template and parts of the container, referred to as parametric user-defined features (UDF), are searched for in the standard library of the PLM system. Copies of the template and UDF models are saved to the workspace of the PLM system, that is dedicated to the configuration process. The content of the workspace after loading all the objects that will be used in the configuration process is shown in Figure 6.

In the next step, the automatic process of generating a parametric $3 \mathrm{D}$ model of the container is started. The template is loaded from the workspace to the CAx session, then the user-defined features are loaded and added to the template (neck, finish and heel). During this process there also occurs multiple, iterative updates of the built model based on the parameters read from the XML file and design rules embedded in user-defined features. The final effect
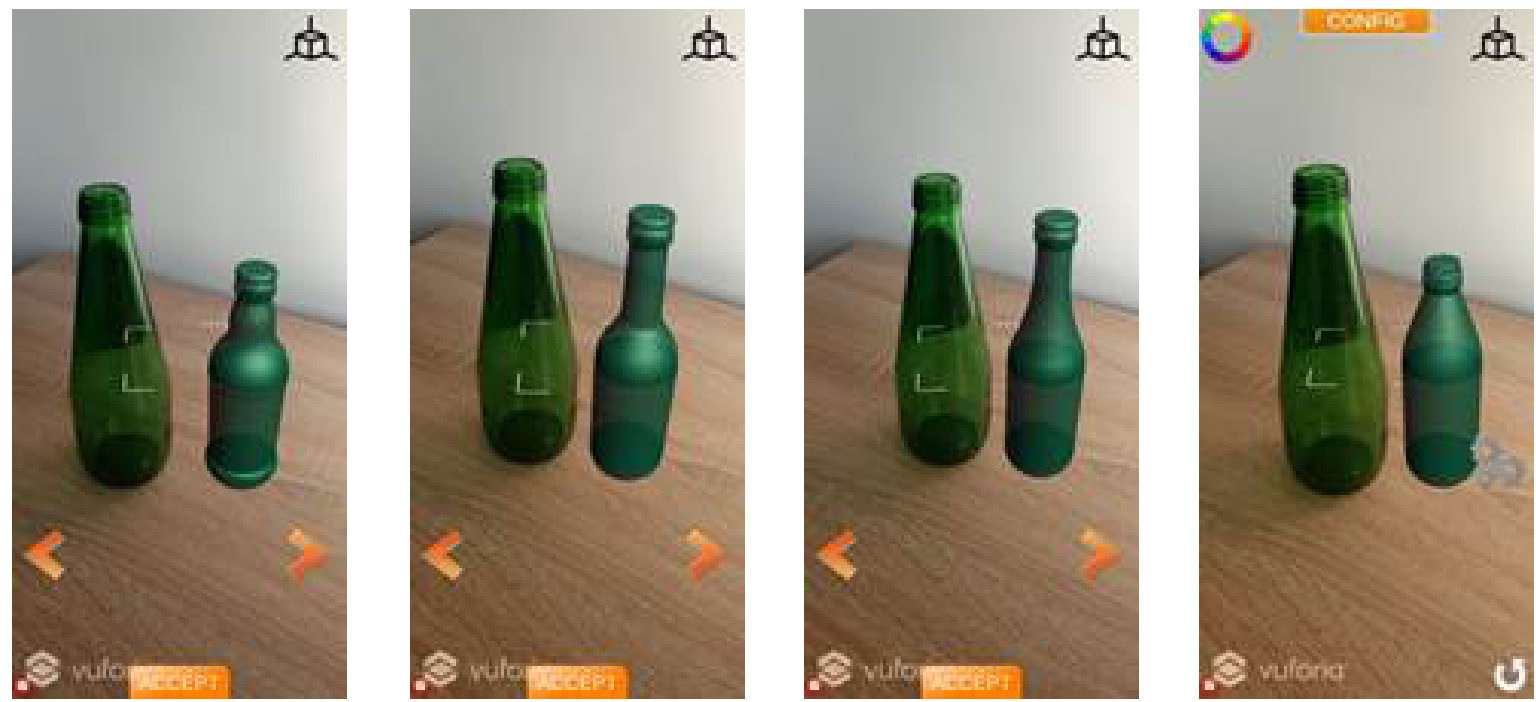

Figure 5. The process of obtaining the initial shape of a 3D model of a glass container in the AR environment

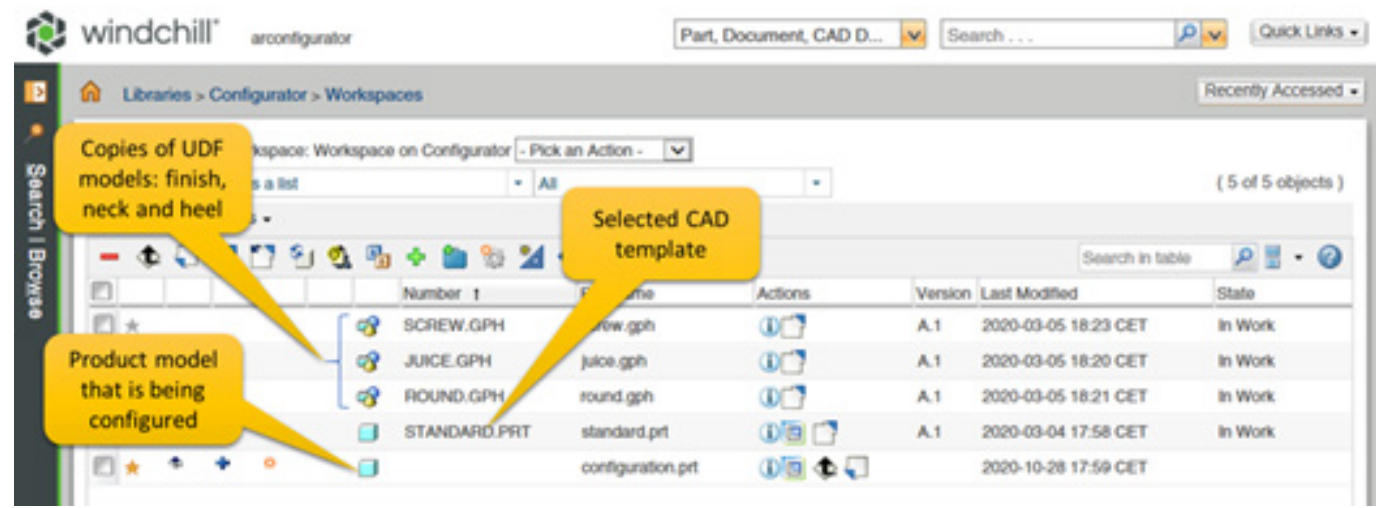

Figure 6. View of the PLM system workspace to which the objects that were used to create the parametric model in the CAx system were copied 
of the parametric $3 \mathrm{D}$ model generation process is shown in Figure 7.

After generating a parametric 3D model, a macro that is run saves it to the lightweight OBJ format. Then, the exported 3D model is sent by the integration module to the mobile application. In the last step, carried out in the mobile application, the transferred model is loaded onto the AR scene and displayed on the screen of the mobile device.

\subsection{Editing dimensional parameters of the 3D model}

In the second phase of the configuration process, one can obtain the exact shape of the created 3D model of the product by editing the selected dimensional parameters. Editing parameters is carried out by entering values in the appropriate fields in a dedicated menu of the mobile application. Once approved, the parametric model update process is initiated. It is very similar to the process described in section 5.3. The entered parameter values are saved to an XML file, and then the file is sent to the server where the PLM system is running. Data read from the file are loaded into the integration module and transferred to the CAx system. The parametric model generated in the previous step is updated to the new parameter values. After the update, the same automated procedure that was used to generate the $3 \mathrm{D}$ model in section 5.3 is repeated (a macro that is run saves the updated $3 \mathrm{D}$ model to the OBJ format, and then it is sent in this format to the mobile application). In the last step, the model updated on the AR scene is displayed on the screen of the mobile device. These steps are iteratively repeated until the desired shape of the designed product is achieved. The activities related to editing the model parameters are shown in Figure 8. Virtual model shape modification performed in order to obtain the desired shape may, in practice, consist of many iterations. For simplicity, the Figure shows only some intermediate steps.

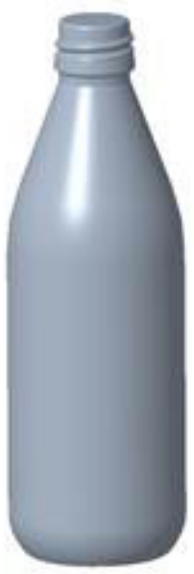

Figure 7. Parametric model of a glass container automatically created in the CAx system based on data sent from the AR application
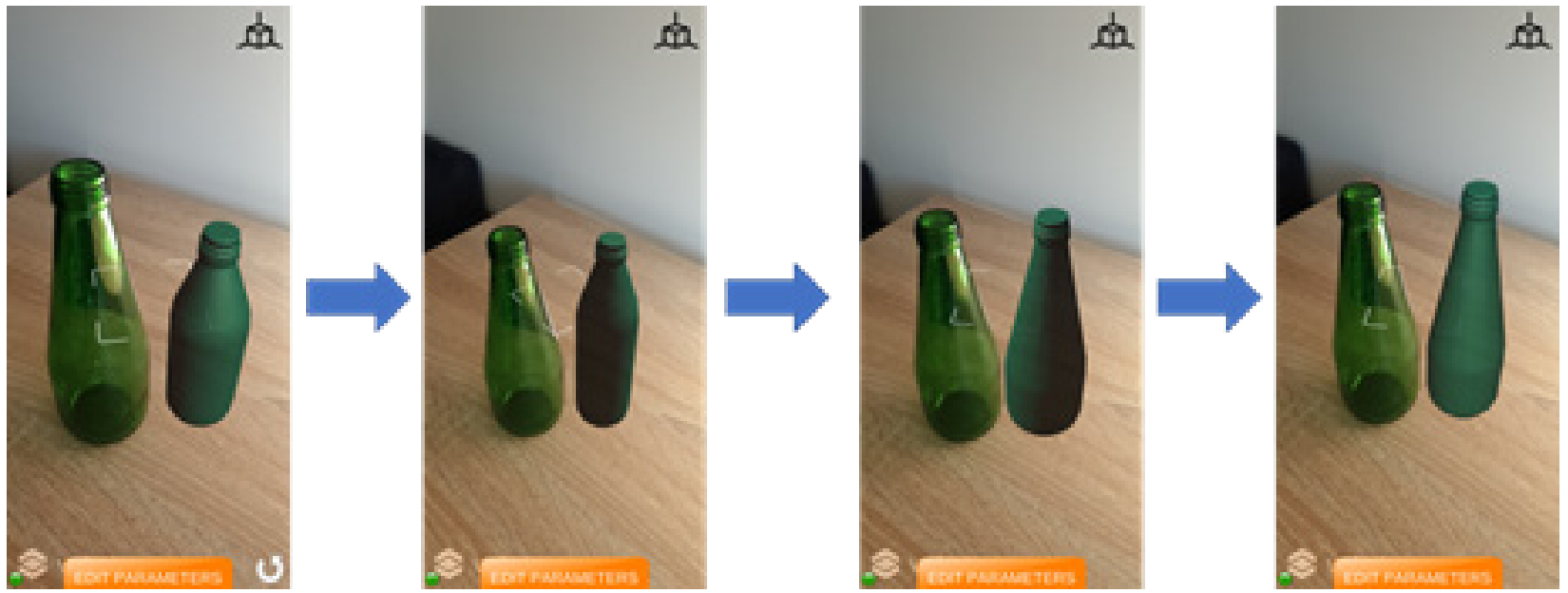

Figure 8. Subsequent (selected) iterations of the virtual model shape modification in the AR envi onment 


\subsection{Saving the created model in the PLM system}

After obtaining the target shape, the model is saved in the mobile application. When saving, it is needed to give a name to the designed product. After doing this, the name of the parametric model, which is in the workspace of the PLM system and in the CAx system session, is changed to the name defined in the mobile application. In the next step, the checkin operation of the parametric model is performed in the PLM system. As a result, the status of the created model is changed to 'checked in.' The model is also copied to the folder defined in the configuration settings in the common space of the PLM system. Once this action is done, the created model is visible to all system users, and the formal process of its validation and approval can begin. Figure 9 depicts the view of the collaboration space.

\subsection{Finishing the process}

After saving the model, a new design session can be started or work in the system can be finished. Ending the operation of the mobile application results in the removal of all existing data from the PLM system workspace. In the next step, the session in the PLM system is closed, and the operation of the CAx system is ended. The final effect of the glass container design process is shown in Figure. 10.

On the left, there is a virtual model of the container in the AR environment, next to the existing bottle. The parametric model created automatically in the PLM system is shown on the right.

\subsection{Assessment of the implemented solution}

This paper presents the results of the research involving proprietary AR system and the commercial PLM system software integration. In its original version, the AR system was developed as an independent solution, which included two components the AR application for mobile devices and the CAx system operating on the server. Communication and data exchange between these components was carried out through an integration module, which also ran on the server. All data, both input, i.e., design templates and parametric user-defined features, as well as output, i.e., the effect of the configuration process, were stored in specific locations, e.g., on local or network drives. Therefore, it was not possible to track the history of changes, implement a formal

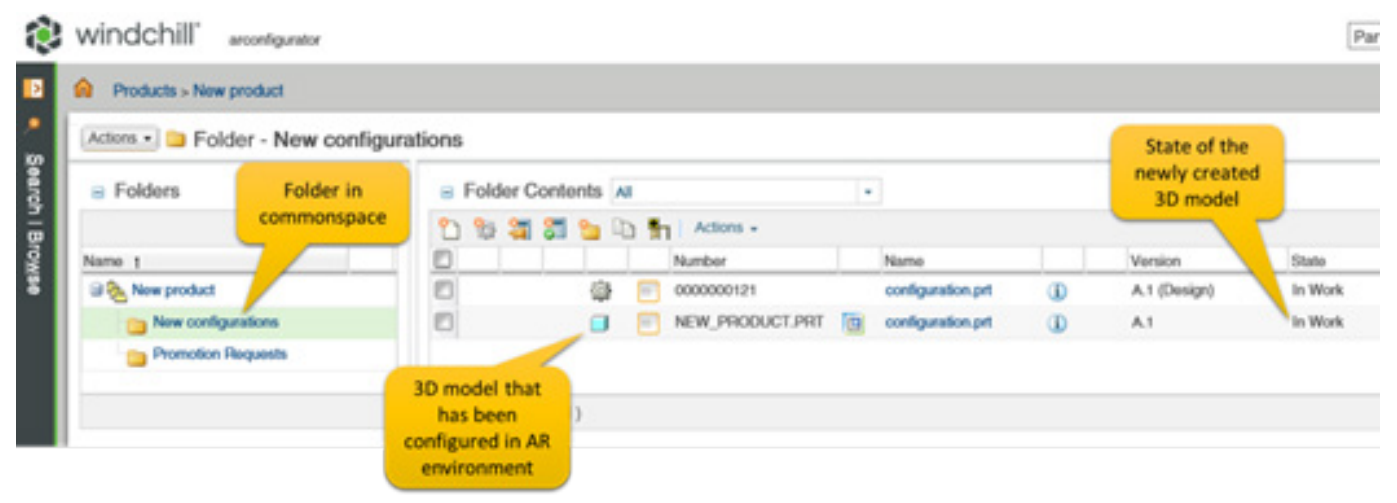

Figure 9. View of the common space after the completion of design acti ities
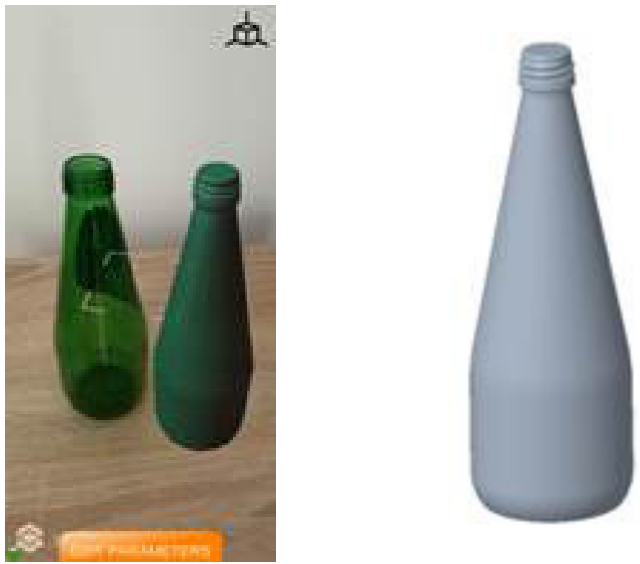

Figure10. The completed virtual model of the container in the AR environment and the corresponding parametric model 
review and approval process, manage states, manage permissions, and easily share virtual models of the products being developed. Equally important, the input data was not secure enough as it could have been accidentally modified, overwritten or even unintentionally deleted. The failure of the disk or server with the data stored was also possible.

The integration developed and presented in this work enables the elimination of all the above-mentioned difficulties and threats. Both the input data and the developed virtual models of products are stored in the PLM environment at every stage of the process. The input data, which are design templates and UDF models, are stored in a dedicated PLM context - in the standard library. All users who participate in the configuration process have access to the data stored in the library. However, editing rights have been restricted and granted only and exclusively to selected users who are authorized to modify and further develop these components. All activities related to the development of parametric models of products with the use of the AR mobile application are carried out in a dedicated workspace. During the configuration process, these data are not visible to other users of the PLM system. Nevertheless, at the end of the configuration process, they are automatically promoted to the next status and copied to the PLM commonspace, so that the design process can be continued immediately. All data in the PLM system are subject to the rules that apply in this system. It is of particular importance to track the history of their modifications, version control, status monitoring and data access protection based on the rights granted. The data cannot be deleted or modified in an accidental and uncontrolled way. Data security in PLM systems is also ensured by a specially designed system and hardware architecture, which eliminates the risks associated with failures or other random events.

During the work related to the development of integration, modifications to the AR system itself were also introduced.

The first modification is related to the change of the marker-based tracking system to a newer solution that allows displaying virtual models without the use of markers. This solution introduces much greater flexibility. In the original solution, the configuration process could not be carried out without markers at all, so it was necessary to print them in the required quality and manage them in such a way that they were available at the time and in the place where the configuration process was carried out. The new tracking system completely eliminates these inconveniences.
However, the markerless tracking system has some drawbacks. Although the surfaces are recognized by the algorithm very quickly, its operation to a much greater extent than in the case of a marker-based solution depends on the lighting of these surfaces. In case of insufficient lighting conditions, the surfaces may not be recognized at all or there may be errors related to the inappropriate scale of the virtual model in relation to the environment. The model may also be displayed in an unstable manner, e.g. its position may be changed randomly, there may be a shaking effect of the model or it may disappear temporarily.

The second modification that was introduced in the AR system during the research was the change of a way the $3 \mathrm{D}$ model configuration process of the container is presented. The original solution, where only one part class was assigned to one dedicated marker, has been replaced with a more convenient solution, where the entire process is carried out in the target context. In the first version, the configuration had to be carried out gradually. First, the user selected individual parts from the available resources using dedicated marker, and then put them together into a complete model on one main marker. Changing the part placed on the model, e.g. the neck, also took place in many stages. First, it was necessary to remove the existing part from the model, then the user selected a new part using the appropriate marker, and then reinserted the selected part into the model. In the present solution, the whole process runs in a very natural way, and the user at every stage of the process performs actions in the target context. All changes in the configured model are presented in real-time.

However, the authors see the possibilities of refining the user interface of the AR application in order to improve ergonomics, better use of the possibilities of touch operation, and to implement gesture control.

\section{Discussion and Conclusions}

This paper presents practical definitions of virtual and augmented reality and virtual factory. The authors highlighted that one of the possible, although not perfect, implementations of the virtual factory paradigm is using modern PLM systems. In these systems, AR and VR technologies can be implemented at the presentation layer level. However, this requires the development and integration of additional services with the PLM system, the purpose of which is to carry out tasks related to data conversion and processing in a specific way (depending on the technology and purpose) so that they can be displayed in 
AR or VR devices.

The paper also proposes a concept of PLM system integration with AR techniques based on generalized PLM system architecture. This concept is based on the assumption that at the level of the application layer integrated with the PLM system, there is a software service by means of which tasks related to AR technologies are performed.

The next part of the paper presents the proposal of the procedure of the technical realization of integration based on the example of a commercial PLM and multi-module proprietary AR systems. The aim and benefits of obtaining a uniform PLM and AR environment have been defined, and additional functionalities that are possible to achieve (e.g. version control, statuses and states management, management of elements used in the configuration process based on the principles of the standards library in the PLM system) have been highlighted.

The last part of the work presents the procedure of the integration of the proprietary AR system with the commercial PLM system. The implemented integration significantly improves the product configuration process in the AR environment. The most important advantages are: ensuring security and a much better-organized data management process (tracking data change history, protection against accidental modification). The PLM environment also facilitates the possibility to implement the design process collaboratively, as the parametric model developed in the AR environment is automatically made available in the common space of the PLM system. The developed integration is a prototype solution, so in order to implement it in the production environment, it is necessary to introduce improvements, especially in the area of code quality of the developed programs. Further research in the area of the tracking process may also be necessary to ensure greater stability of the display of virtual models in the AR environment. Nevertheless, thanks to the developed solution, numerous advantages of implementing AR technology in the PLM environment have been proven and confirmed. The unquestionable synergy resulting from the interaction of these technologies makes it possible to set directions for further research in this area. In the opinion of the authors, one of the most important is the use of both techniques, AR and VR, to support activities in the remaining stages of the product lifecycle, not only in the area of the design process. Another direction, which in the opinion of the authors is worth following, is the use of the AR system developed in [14] in a different than glass containers product domain. The authors also see oppor- tunities to improve the operation of the AR system from a technical point of view. The greatest benefit would be changing the existing communication protocol (that allows the exchange of data between the mobile application and PLM system) to the most popular HTTP protocol. This would enable the use of a universal REST (Representational State Transfer) communication interface [22-23]. As a result, the development of the system in this direction would enable easier integration of the mobile application with other commercial PLM systems.

\section{Acknowledgments}

This paper is an extended version of the original article published in the 41st IFIP International Conference on Advances in Production Management Systems (APMS2020) [12].

\section{Funding}

This research did not receive any specific grant from funding agencies in the public, commercial, or not-for-profit sectors.

\section{References}

[1] S.Mann, Intelligent image processing. Adaptive and learning systems for signal processing, communications, and control. IEEE, New York, NY, United States 2002.

[2] W. Barfield, Fundamentals of wearable computers and augmented reality. 2nd ed., CRC Press, NW Boca Raton, FL, United States, 2015.

[3] M. Januszka, Metoda wspomagania procesu projektowania i konstruowania z zastosowaniem poszerzonej rzeczywistości. $\mathrm{PhD}$ thesis, Silesian University of Technology, Gliwice, 2012.

[4] G. Ghielmini, P. Pedrazzoli, D. Rovere, W. Terkaj, C. R. Boer, G. Dal Maso, F. Milella, M. Sacco. "Virtual Factory Manager for semantic data handling," CIRP Journal of Manufacturing Science and Technology, vol. 6, pp. 281291, 2013. doi:10.1016/j.cirpj.2013.08.001

[5] J. Stark, Product Lifecycle Management (PLM), Product Lifecycle Management (Volume 1). Decision Engineering, Springer, Geneva, Switzerland, 2020, doi: 10.1007/978-3-030-28864-8.

[6] A. Saaksvuori, A. Immonen, Product lifecycle management (third edition). Springer, Heidelberg, Germany, 2008.

[7] J. Duda, Zarządzanie rozwojem wyrobów w ujęciu systemowym. Cracow University of Technology 2016.

[8] W. Terkaj, G. Pedrielli, M. Sacco, "Virtual Factory Data Model," CEUR Workshop Proceedings, vol. 886, pp. 29-43, 2012

[9] T. Tolio, D. Ceglarek, H.A. ElMaraghy, A. Fischer, S. Hu, L. Laperrière, S. Newman, J. Váncza, "SPECIES - Coevolution of Products, Processes and Production Systems," CIRP Annals - Manufacturing Technology, vol. 59, No. 2, pp. 672-693, 2010, doi: 10.1016/j.cirp.2010.05.008

[10] T. Tolio, M. Sacco, W. Terkaj, M. Urgo, "Virtual Factory: 
an Integrated Framework for Manufacturing Systems Design and Analysis," Forty Sixth CIRP Conference on Manufacturing Systems, 2013.

[11] M. Lafleur, W. Terkaj, F. Belkadi, M. Urgo, A. Bernard, M. Colledani, "An Onto-Based Interoperability Framework for the Connection of PLM and Production Capability Tools," pp. 134-145, 2016, doi: 10.1007/978-3-319-54660 $-5 \_13$

[12]J. Duda, and S. Oleszek, "Concept of PLM Application Integration with VR and AR Techniques," in IFIP Advances in Information and Communication Technology, 2020, vol. 592 IFIP, pp. 91-99, doi: 10.1007/978-3-030-57997-5_11, 2020.

[13]J. E. Sienkiewicz, P. Syty, "Architektura warstwowa aplikacji internetowych. Oblicza Internetu,” Conference Proceedings, PWSZ, Elblag (2008).

[14] S. Oleszek, „Metoda wspomagania projektowania naczyń szklanych z zastosowaniem konfiguratora w środowisku poszerzonej rzeczywistości,”. PhD disertation, Silesian Univ. of Tech., Gliwice, 2018.

[15] E. Lavieri. Getting Started with Unity 2018 - Third Edition: A Beginner's Guide to 2D and 3D Game Development with Unity, Packt Publishing Ltd, 2018.

[16]J. Linowes, K. Babilinski, Augmented Reality for Developers: Build practical augmented reality applications with Unity, ARCore, ARKit, and Vuforia. Packt Publishing Ltd, 2017.

[17] PTC Windchill PLM Solutions, "PTC University", https://precisionlms.ptc.com/viewer/course/en/34668550/ page/34668556 [Accessed: 07-Dec-2020].

[18] Vuforia, "Unity User Manual”, https:/docs.unity3d. com/2017.4/Documentation/Manual/vuforia-sdk-overview. html [Accessed: 03-Oct-2020].

[19] Recommended Devices, "Vuforia Developer Library", https://ibrary.vuforia.com/content/vuforia-library/en/ platform-support/vuforia-engine-recommended-devices. html [Accessed: 08-Dec-2020].

[20] Vuforia Fusion, "Vuforia Developer Library", https:/library .vuforia.com/content/vuforia-library/en/articles/Training /vuforia-fusion-article.html [Accessed: 08-Dec-2020].

[21] P. Nowacki, M. Woda, "Capabilities of ARCore and ARKit Platforms for AR/VR Applications," Engineering in De pendability of Computer Systems and Networks, pp. 358370. Edition: 987, Springer, 2020, doi: 10.1007/978-3-03019501-4_36.

[22] H. A. Nguyen, H. Nguyen, H. T. Nguyen, A. C. Phan, and Y. Matsui, "Empirical study on the role of collaboration in new product development in manufacturing companies,” Int. J. Qual. Res., vol. 12, no. 2, pp. 363-384, 2018, doi: 10.18421/IJQR12.02-05.

[23] M. Masse, REST API Design Rulebook. Oreilly and Associate Series, O’Reilly Media, 2011. 